\title{
Content-Dependent Question Generation using LOD for History Learning in Open Learning Space
}

\author{
Corentin Jouault
}

Kazuhisa Seta

Yuki Hayashi

\author{
Osaka Prefecture University \\ jouault.corentin@gmail.com, http://ks.kis.osakafu-u.ac.jp/john
}

(affiliation as previous author)

seta@mi.s.osakafu-u.ac.jp, http://ks.kis.osakafu-u.ac.jp

(affiliation as previous author)

hayashi@kis.osakafu-u.ac.jp, http://www2.kis.osakafu-u.ac.jp/ hayashi/

keywords: question generation, linked open data, semantic open learning space, inquiry based learning, history learning

\begin{abstract}
Summary
The objective of this research is to use current linked open data (LOD) to generate questions automatically to support history learning. This paper tries to clarify the potential of LOD as a learning resource. By linking LOD to natural language documents, we created an open learning space where learners have access to machine understandable natural language information about many topics. The learning environment supports learners with content-dependent questions. In this paper, we describe the question generation method that creates natural language questions using LOD. The integrated data is combined to a history domain ontology and a history dependent question ontology to generate content-dependent questions. To prove whether the generated questions have a potential to support learning, a human expert conducted an evaluation comparing our automatically generated questions with questions generated manually. The results of the evaluation showed that the generated questions could cover more than $80 \%$ of the questions supporting knowledge acquisition generated by humans. In addition, we confirmed the automatically generated questions have a potential to reinforce learners' deep historical understanding.
\end{abstract}

\section{Introduction}

The current state of linked open data (LOD) provides a large amount of content. It is possible to access semantic information about many domains. In this paper, we aim to clarify the potential of LOD as a learning resource. Our hypothesis is that it is possible to generate meaningful content-dependent questions in an open learning space by using the current state of LOD sources.

Questions from the teacher are an important and integral part of the learning and can deepen learners' understanding [24]. More specifically, in the history domain, asking questions itself encourages learners to form an opinion and reinforce their understanding $[11,23]$.

Learners also naturally ask questions themselves during their learning. They contribute to acquiring new information and also to clarifying their misunderstandings. However, they cannot always generate good questions by themselves [22].

Because the quality of the learning is dependent on the quality of the questions [05], asking good questions is im- portant for performing satisfying learning. Learners are required to generate good questions to perform good quality of learning, although when they learn with a teacher, they can rely on the teachers' questions. This is one of the difficulties of learners performing their learning by themselves.

Therefore, research on technology enhanced learning has shown that current learning environments can support learners in self-directed learning by giving inquiry questions [06]. Questions also provide scaffolding which minimizes the difficulties that the learners encounter during self-directed learning [10].

Jouault and Seta proposed a learning environment built to enhance self-directed learning in the domain of history $[13,14,15]$. We plan to embed a question generation function into it for supporting self-directed learning in an open learning space.

Our approach combines a natural language open learning space (Wikipedia) reinforced by semantic information from two LOD sources, DBpedia [03] and Freebase [04]. By integrating these resources, we create a "semantic open 
learning space" that allows the system to understand the semantics of information.

The first issue to be clarified to build the question generation function, which is applicable to an open learning space, is (a) how we build scalable and reliable knowledge resource based on LOD and (b) how we build history domain ontology and history dependent question ontology to generate content-dependent questions.

The second issue to be clarified is whether the quality of the questions generated by the system is sufficient to support history learning. Although we mentioned the importance of questions, we should not provide them to learners if the automatically generated questions are not meaningful enough to deepen historical understanding, because it might have negative effects on the learners. Therefore, we must first of all evaluate the quality of the automatically generated questions themselves before using it.

In this paper, first, we arrange the requirements that should be satisfied by the knowledge resource. Second, we show our method to identify history domain concepts and systemize history domain question ontology. Third, we describe the evaluation on the topic of 'World Wars' comparing the automatically generated questions with questions generated manually by experts to confirm that the automatically generated questions can support learning.

\section{Related Works}

One of the important issues of learning support system is how to realize compatibility of open learning space and content-dependent support. Advantages of self-directed learning, a typical learning style in an open learning space, include a positive effect on learners' motivation because they can follow their interests. Learners also can keep control of their learning, study at their own rhythm by spending more time on their interests and focusing on their weaknesses. One of the disadvantages is that learners in a self-directed learning situation in an open learning space are confronted to a large amount of information not easily manageable for unskilled learners. They can become overwhelmed by the quantity of information and tend to lose their way without control [31].

Thus, to support learners in self-directed learning in open learning space, previous research lead to the creation of systems such as the Navigation Planning Assistant [16], which provides an environment used to describe learners' learning plans and state of understanding to prompt their self-regulation in an open learning space. The limitation of this system is that its support is content-independent due to the difficulty of working with natural language in- formation on the Web. This problem is difficult to overcome.

On the other side, to provide content-dependent advice, learning materials can be prepared in advance in a specific closed domain. This is the case of Betty's Brain [02], which uses a concept map in an environment for learning by teaching, or Kit Build method [09], which provides a knowledge externalization environment for building a concept map using pre-defined kits and supporting the learner during the concept map construction. However, for both systems, the preparation requires a considerable amount of time even for constructing a closed learning space. It is not possible to use the same method in an open learning space because there is too much learning materials. Furthermore, previous research shows that providing manually defined terms representing learning activities, even without providing answers, has the effects of prompting learners' internal self-conversation to understand the contents not explicitly described in a textbook. As a fact, learners could get higher marks for the problems whose answers are not described in a textbook with this method [25, 26].

Our approach tries to build an automatized method that makes content-dependent support compatible with open learning space. In the system using our method, we do not control on the learning materials but the process making them machine understandable can be applied automatically. Therefore, it can be applied to an open learning space, thus, making the system able to generate support depending on the content of the learning materials.

One of the premising methods to support in an open learning space is to ask meaningful questions to learn a specific domain. Inquiry based learning in an open space is recognized as an useful strategy to avoid learners losing their way and disturbing their learning processes [10]. Notable research is Web-based Inquiry Science Environment (WISE) [27], which provides support in self-directed learning. Learners using WISE gather information to answer an inquiry. Learners are trained in designing solutions, debating subjects, and critiquing the resources they learn. However, preparing all the inquiries in advance requires manual processing by specialists.

To lighten this burden, many other research projects aim to generate questions automatically. Most methods can be classified into two categories: syntactic and semantic approaches.

Syntactic approaches alter declarative sentences to turn them into questions. An early example of this approach is work by Wolfe [32]. More recently is work by Heilman [08], which generates factual questions by manipu- 
lating complex sentences. Other notable research includes CEIST system [33], which analyzes sentences and manipulates the syntax trees to generate questions. One of the weaknesses of this approach is that it also generates invalid questions without understanding of typical context of the domain, although it is applicable to wide range of fields.

Semantic approaches try to analyze the meaning of the sentence to generate questions. Research by Agarwal [01] analyzed natural language paragraphs by using discourse cues to generate types of questions: why, when, give an example, and yes/no. Lindberg et al. [19] used semantic role labelling of sentences and generate the questions by using construction rules. Also notable is work by Mazidi and Nielsen [20], which is close to Lindberg' s method but is domain-independent and is also able to provide answers to the generated questions. One of the advantages of the semantic approach is that most of the generated questions are valid based on the understanding of concepts in a domain.

Our method adopts a semantic approach to question generation that uses the LOD and ontologies to create contentdependent question about any historical topic. The main advantage of using LOD to generate questions instead of using natural language sentences is that there cannot be an error in the processing of natural language, thus reducing the number of meaningless questions.

\section{A Use Case of Question Generation Func- tion}

Here, we illustrate our system as a use case of the question generation function to understand its roles to support learning, although we do not focus on its learning effects on learners in this paper.

The question generation function is designed to support learners during self-directed learning of history by being embedded into a learning support system, although it is a general function independent of specific use cases.

Figure 1 shows a screen image of the learning environment where learners can perform their self-directed learning with question generation function. It is composed of four windows:

(a) Question window: It displays a list of questions generated by the system.

(b) Document window: It displays the learning material (Wikipedia document) selected by the learner. The colors of the concepts in the text correspond to dynamic highlighting automatically generated by the system. Orange corresponds to concepts related to the concept associated to the Wikipedia document. Green corresponds to concepts known by the learner. All other concepts are colored in blue.

(c) Answer window: Learners use this window to answer their active questions selected in (a). They can write their answers to deep questions (described in Section $4 \cdot 4$ ) in natural language.

(d) Concept map window: It is used for the learner to manage the concept map. The display of the concept map is built for history learning. The concepts in the middle are events. Events are represented on an automatically generated timeline. The start and end date of each event is displayed in gray inside the concept. The size of each event node depends on the length of the event. Other concepts are colored in blue. The lines between two concepts are relations with the type of relation written at the center of each line. Learners represent their answers to shallow questions (described in Section $4 \cdot 4$ ) by adding relations to the concept map.

When learners use the system, they first set a main topic for their learning, e.g. World War I (WWI), World War II (WWII), Roman Empire, etc. Then, a document in the Wikipedia about the topic appears in the window (b). When learning from the document, learners externalize their knowledge by building the concept map in window (d). For building their concept maps, they can select clickable concepts in the documents (Wikipedia pagelinks) to add them to the concept map. Thus, the system can also understand the contents of the learner's concept map.

The system can detect errors in the learner's concept map by checking the differences with its own concept map. The system is also able to identify which questions are answered by the learner. The system has a potential to manage errors in several ways to make learners aware of their own errors. The details are out of focus in this paper.

Then, at any time they want, they can request questions from the system by clicking the "Generate Questions" button in window (a). The system generates a list of questions that can direct their learning activities. The learners then choose a question they find interesting, and continue their learning to answer it.

In history learning, not only in self-directed learning but also in classroom learning, it is important for learners to build their own image of history [12]. It requires not only memorization of basic information but also knowledge integration activities for learners to interpret the social background at that time period. Giving suitable questions in the contexts of individual learners' states have an effect of making learners aware of the importance of acquiring 


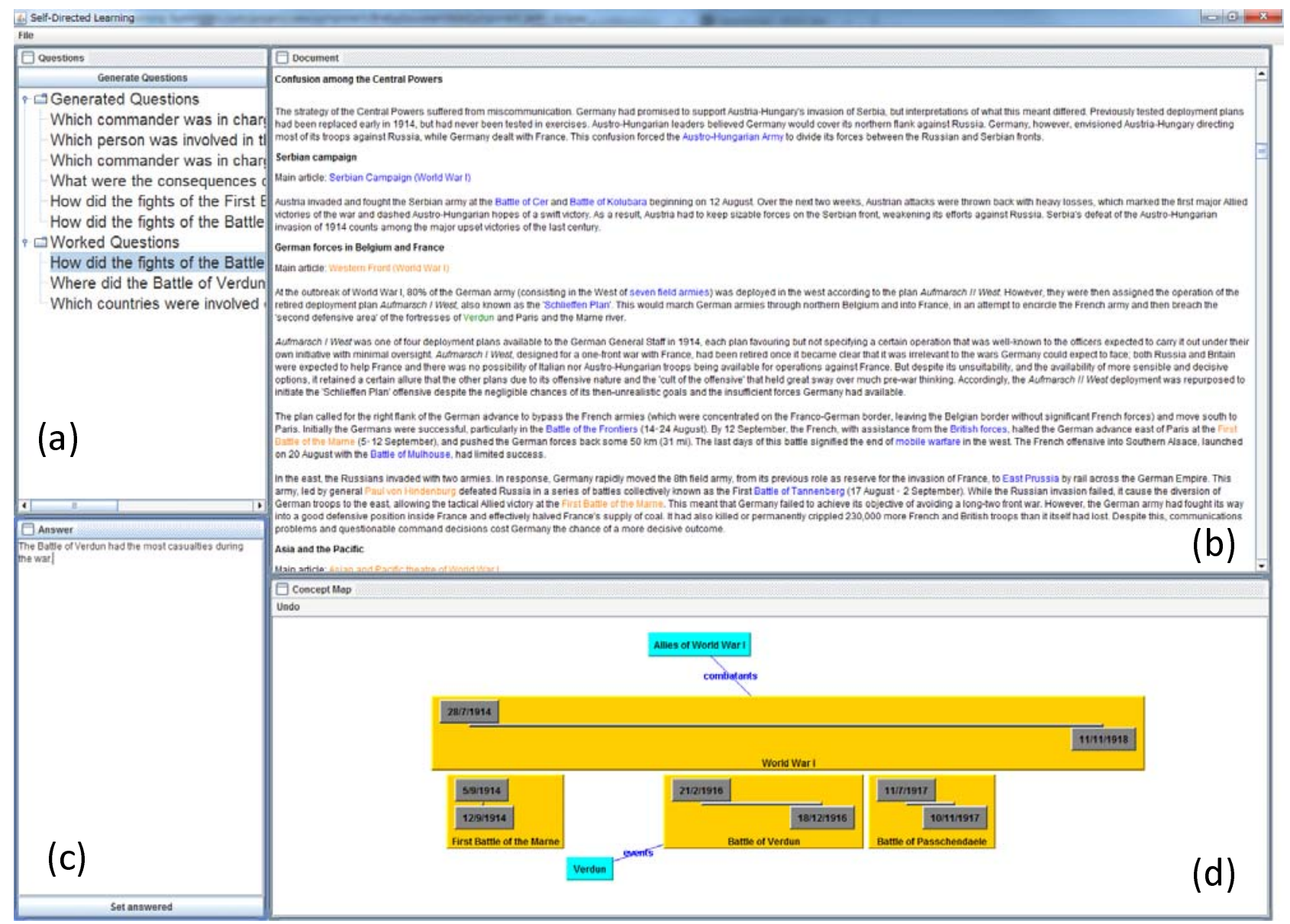

Fig. 1 System interface

new knowledge and to integrate it into their knowledge. The system generates questions adapted to the learner's understanding states represented as the learner's concept map. In order to realize the adaptability of the questions, the system builds its own concept map (Fig. 2) based on LOD to find out the target knowledge to ask by comparing it with the concept map of the learner (Fig. 1 (d)). The system's concept map contains not only all the concept instances and relation instances in the learner's concept map but also more semantic information about them. Thus, the system focuses on the differences between the two concept maps to generate questions adaptively for individual learners' states.

The difference between the two concept maps includes a lot of concepts and relations that the learner did not study yet. Thus, to find suitable targets for the questions, the system adopts a simple ranking algorithm like page ranking. It simply considers a factor of popularity i.e. how many other concepts refer to the concept.

Figure 2 shows a part of the concept instances and semantic relation instances among them which make the system able to understand the chronology including the context. The concepts colored in pink are only in the system's concept map. In chapter 4, we describe the construction process of the system's concept map.

The aims of embedding the function in the system from the viewpoint of learning objectives are two-fold:

(A) Support the development of the domain understanding of the subject to be learned. The system supports learners to help them learn the important historical events.

(B) Support the development of learning skills for selfdirected learning of history. The system helps learners to become aware of the important points during the study of a historical period.

Regarding objective A, the questions provide support to help learners not only memorize historical events but also understand them. In history learning, an understanding of chronology is necessary [30]. Chronology is defined by Smart [28] as "the sequencing of events/people in relation to other and existing knowledge of other, already known, events/people." Learning history is not only remembering a series of facts. Learners need not only to know events, but also to understand their context.

In our system, the learner can represent the context information in the Fig. 1 (d). The purpose of the concept 


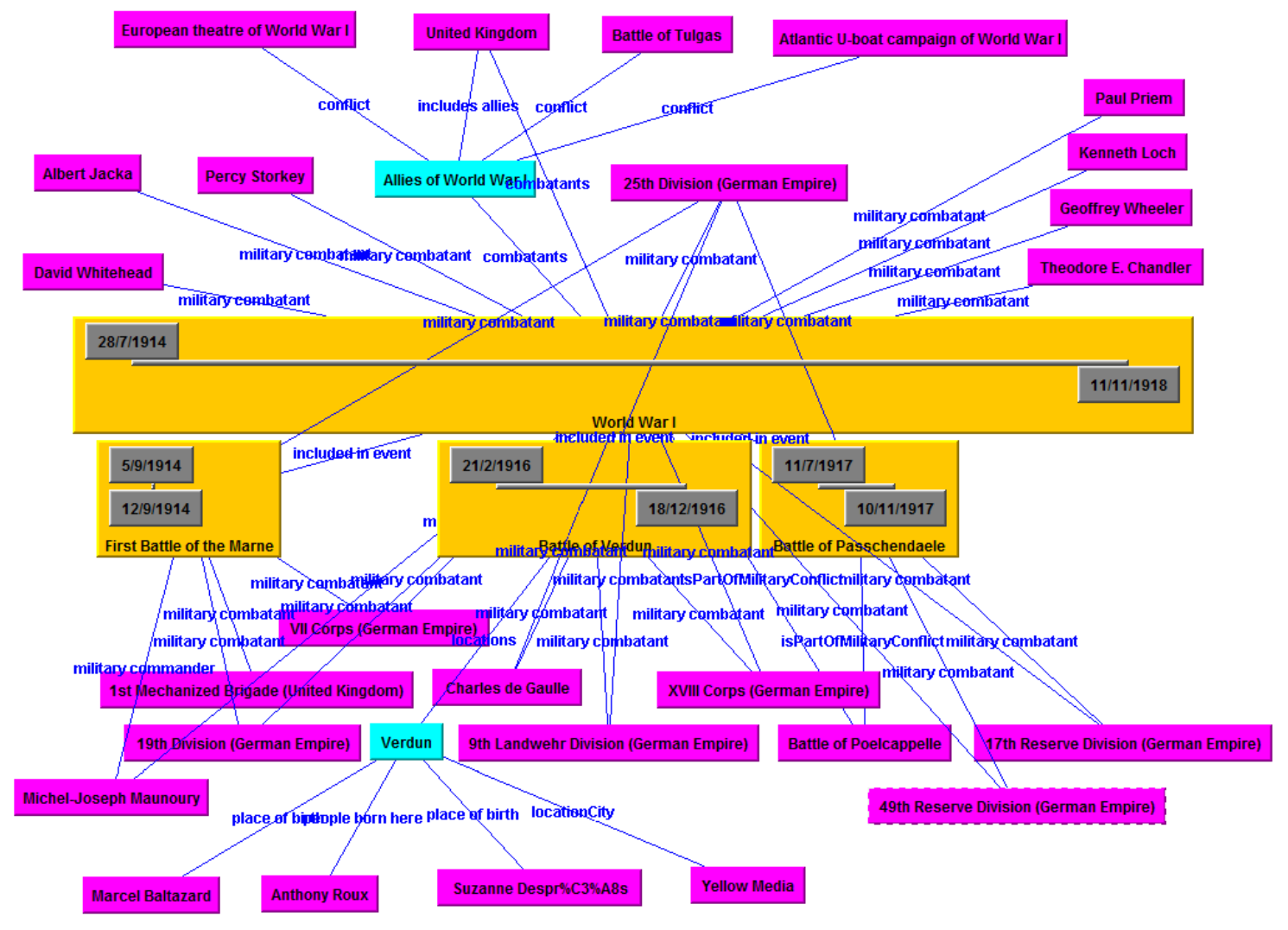

Fig. 2 System's concept map

map window is to make learners describe their understanding explicitly. Research showed that building a concept map deepens the understanding of learners [21]. Building a chronology also reinforces learners' historical understanding [30].

Regarding objective $\mathrm{B}$, we expect questions to have an effect of making learners become aware of the importance of setting objectives in self-directed learning. In our system, learners need to set a learning objective by choosing a question before performing learning activities. Then, learners need to gather information to be able to answer the questions. Repeating the process prompts learners to raise awareness of the importance of inquiry based selfdirected learning of history.

Many researchers pointed out that inquiry based learning has a positive effect in training learning skills, especially self-regulated learning skills in open space learning $[10,27,34]$. Thus, iterating above activities can be expected to improve self-regulated learning skills.

The system we developed is a client-server application. The system uses a mirror server loaded with dumps from LOD (DBpedia and Freebase) to fasten the data gathering process. The client is implemented as a Java application. The client requests data from the server using the server's SPARQL endpoints. The client uses the data requested to generate the system's concept map and the questions.
The next chapter describes how the system generates the questions.

\section{Question Generation Method}

To develop a meaningful question generation function in a historical learning domain, we need to build a scalable and reliable knowledge resource containing both machine understandable and human readable learning materials. Then, we need to build a history domain ontology and history dependent question ontology to generate meaningful questions for history learning.

\subsection{Building integrated LOD}

The problem to be solved is to build a reliable knowledge resource for history learning that has both semantic information and natural language information. Natural language information is required for learners to use as learning materials. The requirements for the knowledge sources are:

(a) Unity: The semantic information should represent the contents of the natural language documents as much as possible.

(b) Scalability: The natural language and semantic information should cover most historical important events that the learners may want to study. Because our sys- 
tem is used for self-directed learning, they set their learning topics and learn freely by themselves, e.g. learning about WWI, the American Civil War, etc.

(c) Reliability: The semantic information represented should be reliable enough to provide meaningful concept instances and context information to learners, especially about important concept instances.

Regarding (a), for the natural language learning materials, we selected Wikipedia because its fast evolution and growth give reliable information about a huge number of topics. In the system, Wikipedia documents are shown as learning materials in Fig. 1 (b). In addition, if errors are present, they are corrected with time. An advantage of selecting Wikipedia is that two semantic knowledge resources (DBpedia [03] and Freebase [04]) are available. Both of the DBpedia and Freebase projects aim to create a semantic copy of the knowledge on Wikipedia. Thus, the requirement of (a) is satisfied by using Wikipedia and its semantic resources.

Regarding (b) and (c), we built a semantically enriched resource by combining the above two semantic resources to give meaningful questions that follow learners' individual interests on their learning topics.

The main difference between the two projects is:

- DBpedia's information is automatically extracted from Wikipedia; it deals with all of the topics in Wikipedia. It covers even minor topics as opposed to Freebase, while the quality of its information is not as high as the human made semantic information in Freebase.

- Freebase's information is provided by humans; the quality of information is higher than that of DBpedia. While it has rich semantic information about major topics, the semantic information about minor topics, but important for advanced learners, is poor.

We understand that DBpedia and Freebase have different characteristics of scalability and reliability. Fig. 3 shows an example of the relation instances defined for two concept instances: "First Battle of the Marne" and "1st Army (German Empire).” These concept instances appear in red. The top part of the figure shows the graphical representation of the relation instances defined for the two concept instances for Freebase (on the left) and DBpedia (on the right).

The first concept instance "First Battle of the Marne" is an important event during WWI. Thus, a lot of relation instances have been defined on Freebase. DBpedia also has relation instances, but not as many as Freebase. On the other side, the second concept instance "1st Army (German Empire)" is minor for most learners, although advanced learners might have interest. For this concept instance, the advantage of DBpedia is particularly visible since Freebase did not define any relation instances about it.

By following the above consideration, we developed a method to satisfy the (b) Scalability and (c) Reliability requirements that combines the two semantic information resources. The bottom part of Fig. 3 shows the combined relation instances with color depending on their provenances. The system embeds the combined and integrated information as its own concept map (as shown in Fig. 2).

By integrating two semantic information resources, we get semantic information that includes the major concept instances with reliable rich semantic information most learners should learn and that also covers minor concept instances that might be of interest for advanced learners. It still maintains the relations with Wikipedia documents, thus satisfying the (a) Unity requirement.

\subsection{Building the history domain ontology}

To enable semantic processing for the integrated data, we need to build an ontology for historical concepts and relations. We, however, need to consider that both DBpedia and Freebase have respective original class and type definitions.

- DBpedia: It has an ontology which has been manually created based on the most commonly used infoboxes within Wikipedia.

- Freebase: It does not have hierarchically organized ontology. It defines types for respective topic categorizations by domain. The organization by domain is designed to make it easier for users unfamiliar with ontology to understand.

Because DBpedia ontology specifies the hierarchy of concept classes, we simply used its hierarchy and mapped the Freebase types to the DBpedia classes by defining equivalents. The advantages are:

(i) The data extracted from DBpedia can be easily applied to the ontology.

(ii) Even the data extracted and integrated from Freebase gets semantically enhanced by referring to the ontology.

Figure 4 shows a portion of the history domain ontology. Here, we mainly describe the concept class definitions, although we also specify relation classes the same way as concept class definitions.

In the top left, the types in each domain defined in Freebase are shown, e.g. the type 'fr:military_conflict' (F:C1) and 'fr:military_person' (F:C3') are in the 'fr:military' domain. 


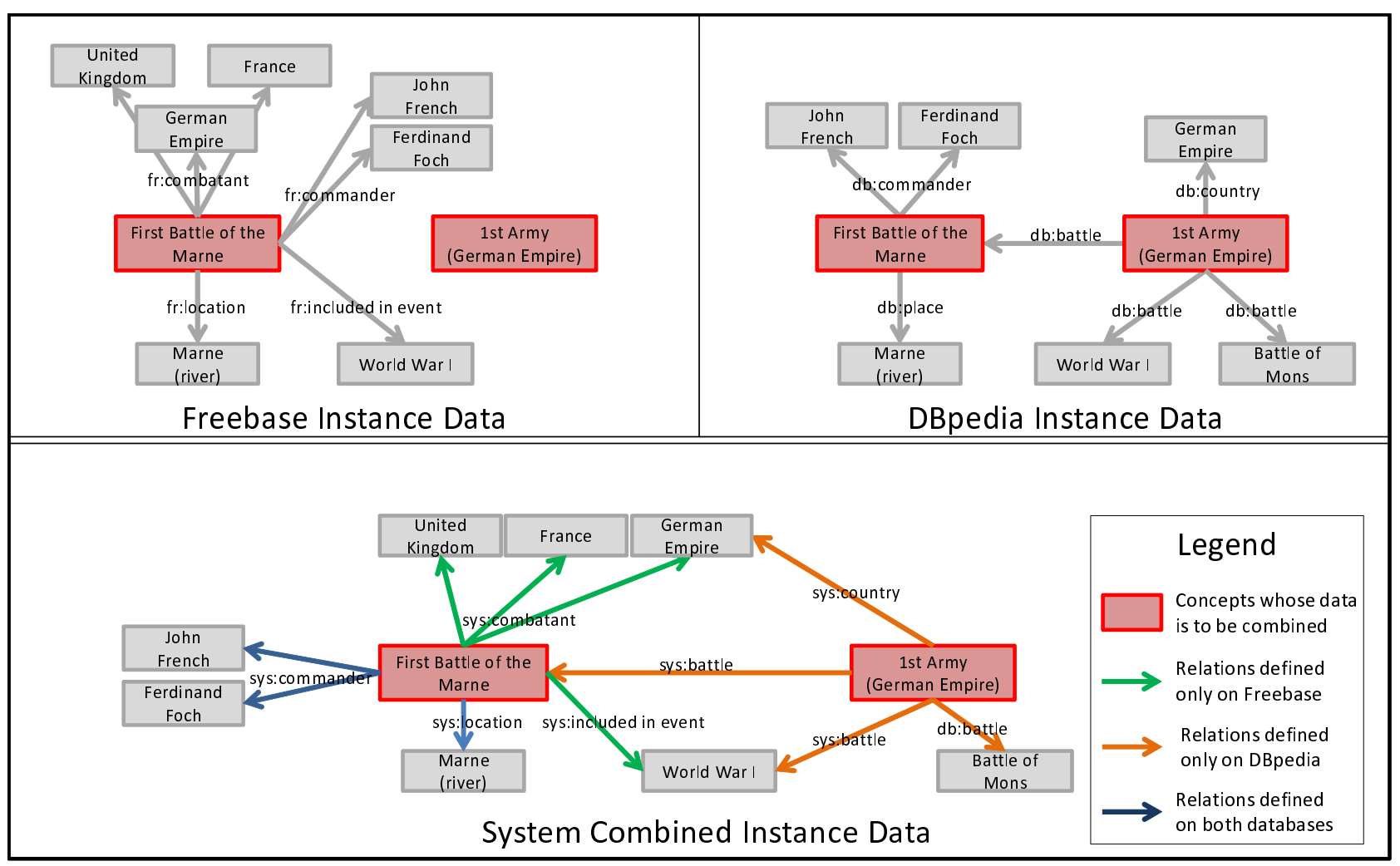

Fig. 3 Combination of Freebase and DBpedia information for two concepts (Concept map built by the system)

The top right shows classes defined in the DBpedia ontology and their hierarchy, e.g. it specifies that the class 'dbpedia-owl:MilitaryConflict' (D:C1) is a sub-class of the class ‘dbpedia-owl:Event' (D:C0), and both classes 'dbpediaowl:Person' (D:C3) and 'dbpedia-owl:Organisation' (D:C4) are a subclass-of the class 'dbpedia-owl:Agent' (D:C2).

The bottom shows a hierarchy of concept classes defined in the history domain ontology. Their hierarchy is adopted from that of DBpedia, e.g. it specifies that both of the concept classes 'Person' (S:C3) and 'Organization'

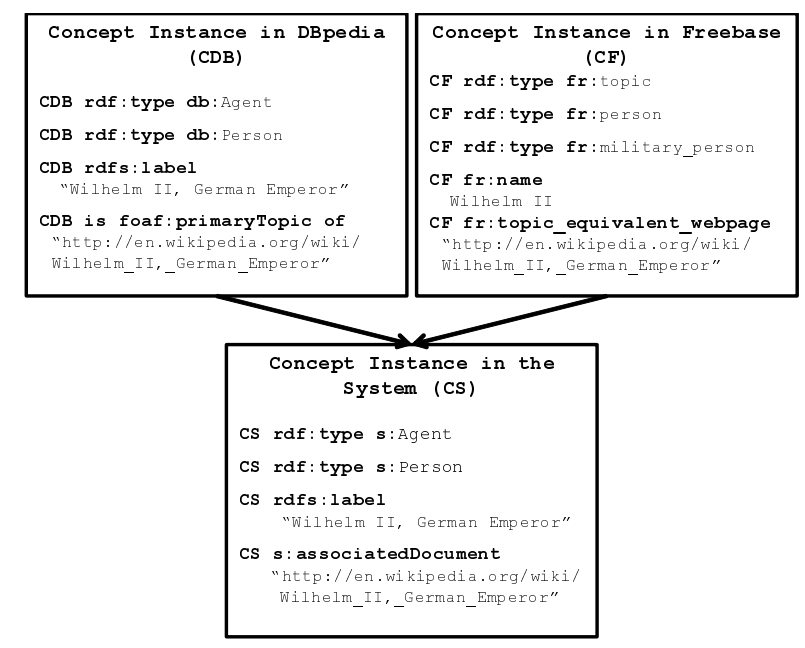

Fig. 5 Internal representation of Wilhelm II in Freebase, DBpedia, and their information merged in the system
(S:C4) are a sub-class of the concept class 'Agent' (S:C2). Furthermore, the equivalents for both semantic resources can be seen under each concept class definition, e.g. the types 'dbpedia-owl:Person' (D:C3), 'fr:person' (F:C3), and 'fr:military_person' (F:C3') specified as the equivalents under the definition of 'Person' (S:C3). According to this definition, the data integrated from a concept instance of 'fr:military_person' (F:C3') and a concept instance of 'fr: person' (F:C3) on Freebase are both dealt as a concept instance of 'Person' (S:C3) in the system.

It also specifies how the system merges the data of two concept instances as an integrated concept instance, even if the system can judge the sameness of the two concept instances by referring to the Wikipedia URI.

Figure 5 shows an example of an internal representation of combined concept instances about "Wilhelm II" based on the definition of concept class 'Person' (S:C3).

Furthermore, the missing concept class or type information is complemented, e.g. when gathering information about the concept instance "Central Powers", DBpedia missed the information that it is an instance of the class 'dbpedia-owl:Organisation' (D:C4), while on Freebase, "Central Powers" is given the type 'fr:organization' (F:C4). By specifying that the class 'dbpedia-owl:Organisation' (D:C4) and the type 'fr:organization' (F:C4) are equivalent under the definition of the concept class 'Organisation' (S:C4), the information can be complemented in the 


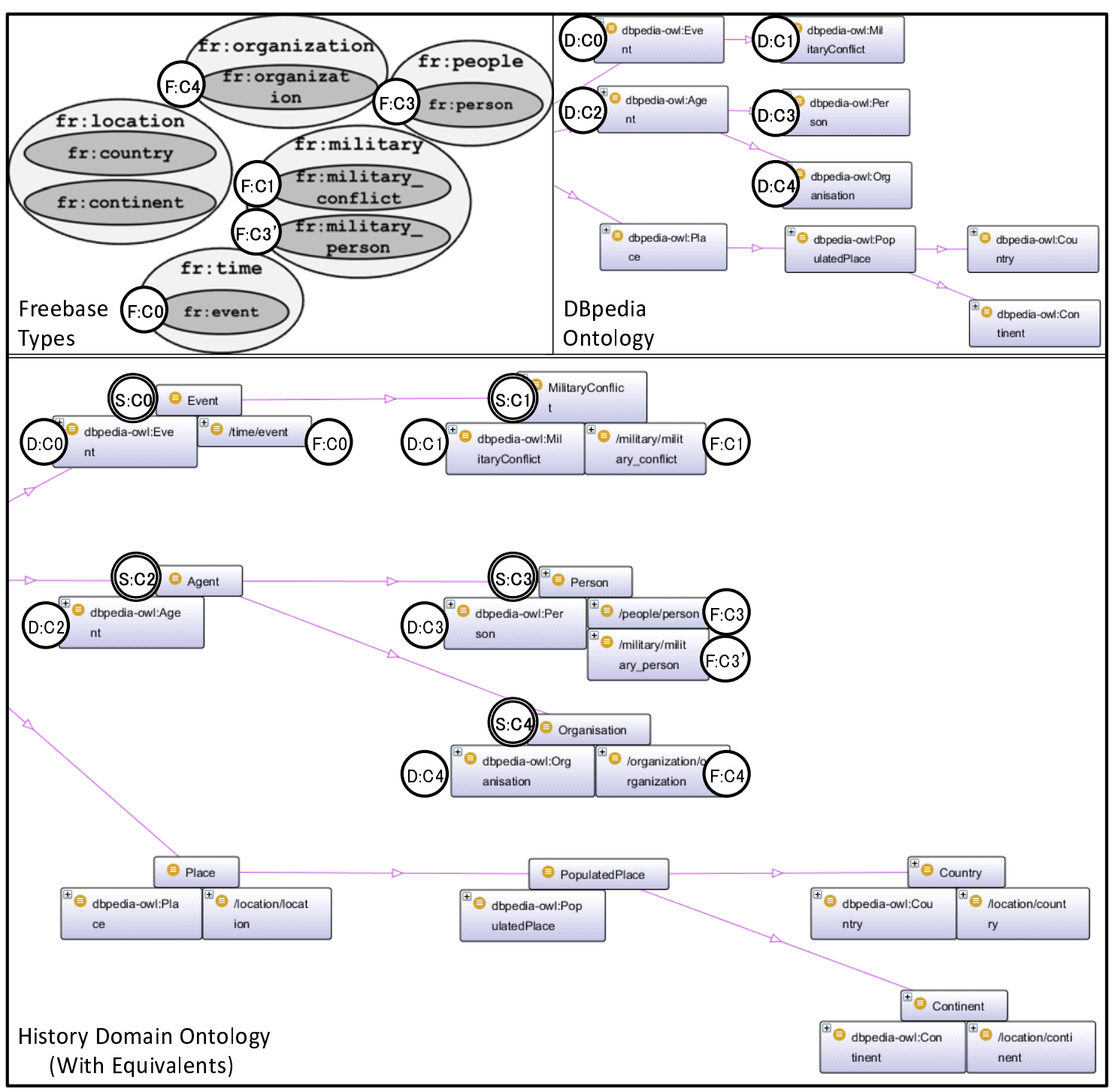

Fig. 4 History domain ontology linking Freebase and DBpedia

combined information by referring to the concept definition of 'dbpedia-owl:Organisation' (D:C4).

For building the history domain ontology, the way we identify enough valid concept (relation) classes related to history and how we set appropriate equivalents are important issues, especially because of the huge size of the DBpedia ontology (currently, 685 classes and 2,795 different properties). Our method to find history related concept (relation) classes and to set appropriate equivalents is simple and partially automatic and the approach is based on real instance data:

(i) First, we identified a huge number of history related pairs of concept (relation) instances from the two semantic resources, each of which shares the same URI, by referring to Wikipedia categories of history.

(ii) Then, the concept (relation) types for both Freebase and DBpedia set for the concept (relation) instances were gathered (the classes with the same label are set as equivalents automatically).

(iii) Finally, we manually associated the equivalent of classes without equivalents and checked the automatically set equivalents.

In the current version of the history domain ontology, we specify 121 concept classes and 282 relation classes.

\section{$4 \cdot 3$ Building the history dependent question ontology}

The system can understand the semantic information to create questions that can support history learning. Furthermore, the system requires an understanding of a meaningful question's structure to generate meaningful history domain questions.

To understand the structure and function of a question, we refer to Graesser's taxonomy [07] to build an ontology for the history domain. This taxonomy describes domainindependent question types that are meaningful to support learning. This taxonomy is used by many research 


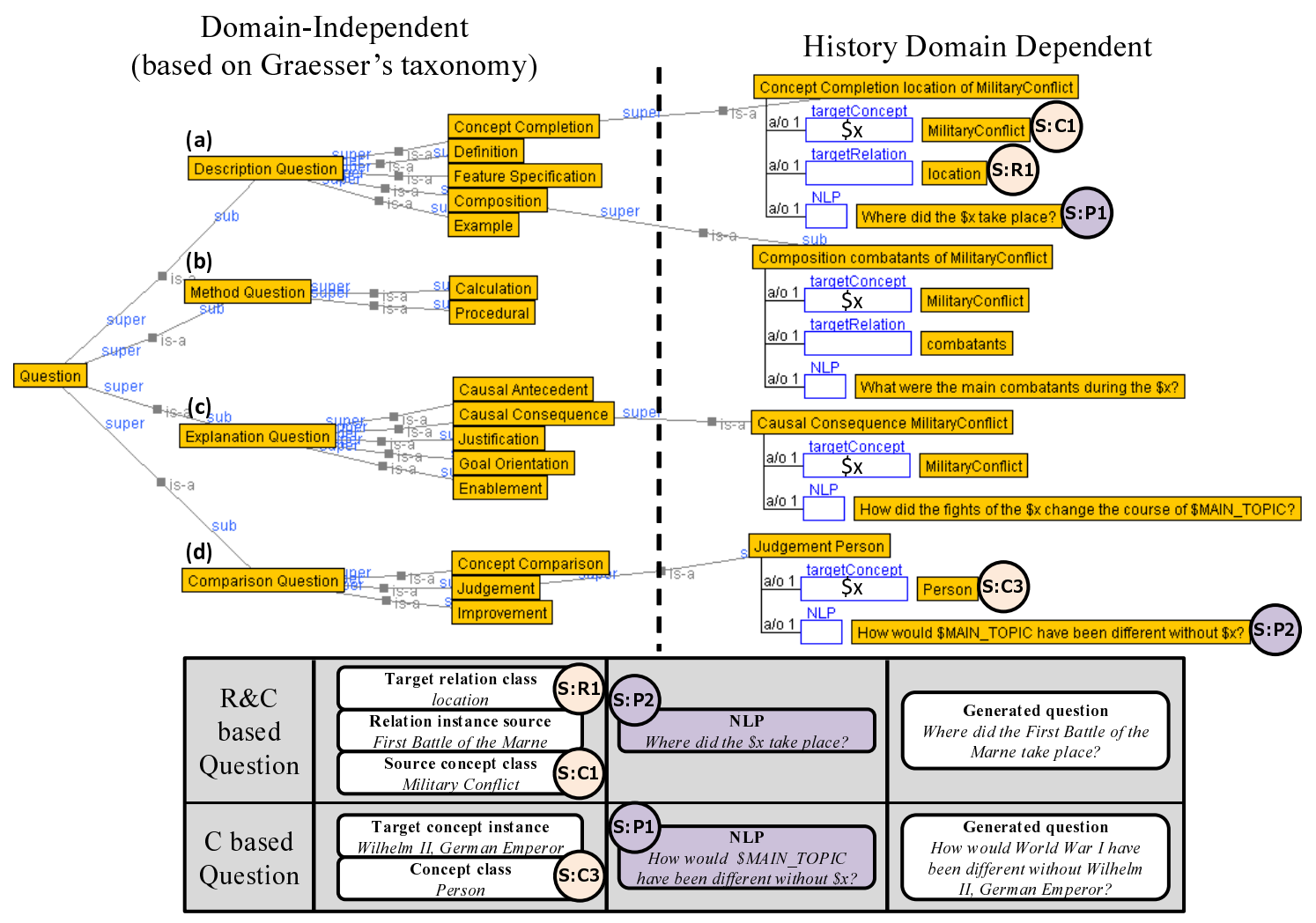

Fig. 6 History dependent question ontology and examples of natural language question generated

projects $[17,18]$.

Figure 6 shows the history dependent question ontology and examples of the natural language questions generated. The top part of the Fig. 6 shows the History Dependent Question Ontology (HDQ Ontology) which is divided in two parts. The left part shows the definition of the domain independent question concept classes based on Graesser's taxonomy. The right part shows the history domain question concept classes that we defined.

The HDQ Ontology specifies that there are four major categories of questions, i.e. (a) 'Description Question', (b) 'Method Question', (c) 'Explanation Question' and (d) 'Comparison Question'. Furthermore, more specialized question concept classes are defined, e.g. 'Concept Comparison' question, 'Judgement' question and 'Improvement' question are defined as a subclass of the class (d) 'Comparison Question'.

History domain question concept classes are organized hierarchically under the domain independent question concept classes, e.g. it specifies that the history domain question concept class 'Concept Completion location of MilitaryConflict' is a subclass of the domain independent question type 'Concept Completion' which is a subclass of the category (a) 'Description Question'.

Each definition of the question concept class in the HDQ Ontology specifies the relation among the concept (rela- tion) classes specified in the history domain ontology and the natural language patterns (NLP).

Each NLP specifies a template of a natural language question for each question concept class and is used to generate the natural language text of the question. For example, the history domain question concept class 'Concept Completion location of MilitaryConflict' associates the concept class 'MilitaryConflict' ( $\mathrm{S}: \mathrm{C} 1)$, the relation class 'location' (S:R1) and the NLP “Where did \$x take place?" which means that, to create the natural language question, the \$x marker is replaced by the 'label' of a concept instance of 'MilitaryConflict' having a 'location' relation instance.

By defining history dependent question concept classes based on Graesser's taxonomy and associating history dependent concept classes with natural language patterns, the system can generate history dependent questions in natural language. Currently, the HDQ define 28 history domain questions.

Based on the definition, the system generates two kinds of questions:

(A) $\mathbf{R} \& \mathbf{C}$ based Question: Relation and Concept based Question. These questions are generated using the history domain question concept classes of the history dependent question concept classes under the question category (a) that require a concept instance 
with a relation instance. The answer to this type of question is identified based on a triple described explicitly in a concept map built by the system.

(B) C based Question: Concept based question. These questions are generated using the history domain question concept classes of the history dependent question concept classes under the question categories (b), (c) and (d) that require only a concept instance. Each of these questions asks even about information not explicitly described.

The table at the bottom of Fig. 6 shows examples of questions for $\mathrm{R} \& \mathrm{C}$ based question (first line) and $\mathrm{C}$ based question (second line). In both cases, the natural language questions are generated by filling the history domain concept instance and relation instance which satisfy the constraints of a question concept class, into the NLP. For example, in the case of the $\mathrm{C}$ based question of Fig. 6, the NLP "How would \$MAIN_TOPIC have been different with $\$ x$ ?" is filled by replacing the $\$ \mathrm{x}$ and $\$$ MAIN_TOPIC markers by respectively the name of the concept instance "Wilhelm II, German Emperor" and the name of the main topic of study, in this case "World War I."

In the actual learning scenario, to limit the number of questions provided to the learner, the system identifies the target concept (relation) instances of the questions by comparing the concept map built by the learner and the concept map built by the system with the semantic information. These targets are identified by checking the information missing from the learner concept map.

\section{$4 \cdot 4$ Function of the questions in history learning}

For history learning, Riley [23] pointed out that the questions can be separated into two groups depending on their function:

(A) Shallow question: These are simple questions that can be answered easily. If learners do not know the information necessary to answer the question, they can usually find it explicitly described in the documents. Shallow questions are the kind of questions that would typically be asked in a basic knowledge test e.g. "Where did the First Battle of the Marne take place?" The shallow questions only lead learners to learn about basic knowledge.

(B) Deep question: These are questions difficult to answer and requiring the learner to think about the learnt information. Deep questions are the kind of questions that would typically be asked for an essay writing test e.g. "How would World War I have been different without Wilhelm II, German Emperor?" More examples of deep questions appear in Table 3 in the next chapter.

Regarding the shallow questions, the $\mathrm{R} \& \mathrm{C}$ based questions can be considered shallow questions because currently the relation instances (predicates) described in the current LOD contain only basic relations for history learning. Question generated based on "Description Questions" (Fig. 6 (a)) of Graesser's taxonomy correspond to the shallow questions. It supports learners in building a basic knowledge of the topic.

Regarding the deep questions, the $\mathrm{C}$ based questions can be considered deep questions because the knowledge required to answer is specified in the definition of the HDQ Ontology. Question generated based on the categories Fig. 6 (b), (c) and (d) of Graesser's taxonomy correspond to the deep questions.

Bransford et al. [05] pointed out the importance of giving domain-dependent meaningful question e.g. when learning history, inquiry about "who wrote this document, and how does that affect the interpretation of events?", whereas, when learning physics, inquiry about the underlying physical principle at work. While learning about an historical topic, it is necessary for learners to learn basic issues about persons, events, etc. but only memorization about basic topics does not always deepen their historical understanding.

To deepen their historical understanding, although it is hard to define what historical understanding is, learners need to actively develop their knowledge by themselves through integrating their basic knowledge and questioning to build their own image/interpretation [12]. Furthermore, the history professor, appearing in next chapter as evaluator, emphasized the importance for learners to build their own opinion in history learning, even about topics without any definite answer. He pointed out that an important learning activity in history is to imagine the situation surrounding an event and the social forces influencing that situation.

Of course, if the questions are not valid to deepen historical understanding, we should not provide them to learners. Therefore, we consider that evaluating the quality of the automatically generated questions is essential.

We assume that both shallow and deep questions can be generated by the system. The evaluation described in next chapter aims to validate this assumption. 


\section{Evaluation of Quality of Questions}

\section{$5 \cdot 1$ Evaluation method}

\section{$\S 1$ General evaluation setting}

In general, it is difficult to simply compare manually generated questions with automatically generated ones because they are not generated in the same conditions or objectives; a typical difference is that the system generates questions according to an "individual" learner's concept map, whereas authors of a textbook do so according to "assumed learners (specific or general learning objectives)."

In this paper, we adopted SparkNotes as a resource of questions that is used by a huge number of learners. Then, we asked a history professor to compare the quality of questions according to his criteria for evaluating shallow and deep questions. More detailed information about the evaluation such as the topic, SparkNotes, and the evaluator is as follows.

- Topic: WWI and WWII. Our method is specific topicindependent although the system embeds history domain dependent elements. Thus, the current version of the system can generate history domain questions for any topic e.g. the Egyptian Civilization, the Roman Empire, etc. However, we might need to add definitions of questions and natural language patterns in the question ontology to adapt, even though our framework can accept such extensions without any changes of question generation system. Thus, we have to carefully address this issue with the combination of the set of definitions in question ontology and historical topics. Based on this recognition and to raise the reliability of the results, we judged that it is more adequate to choose the topics in the field to which the same set of definitions of question ontology can be applied.

- Source of human generated questions: SparkNotes [29]. This is a popular website, whose target users are mainly junior high-school and high-school students that provides learning materials, quizzes and essay questions in many domains. Around 20 million unique users from all over the world access the website each month. Thus, it is recognized as a meaningful website for history learning and gives fundamental questions to support learning. The questions for our experimental study were taken from the multiple choice quiz and essay questions. We used all of the 58 questions about WWI (58: WWII) in SparkNotes. Then, they were manually separated into 38 shallow (31: WWII) and 20 (27: WWII) deep questions by identifying the 38 (31: WWII) shallow questions with an answer written explicitly in the learning materials. The remaining
20 (27: WWII) questions not having a definite answer, like essay questions, but contributing to deepening history understanding were categorized into deep questions.

- Evaluator: A history professor at a university with over 20 years of history teaching experience.

For this evaluation, it is essentially not possible to evaluate objectively the quality of questions according to some common objective criteria. Only subjective evaluation by human experts, based on their respective criteria, is possible.

It is difficult to objectively generalize the evaluation results. Respective results conducted by human experts are meaningful even if they might have contradictions. Results from different experts should not be merged because they are built on different subjective criteria.

In our experimental study, while only one reliable expert with considerable experience of history teaching conducted this evaluation, his opinion is valuable because it is built on a strong knowledge of historical topics, history learning strategies, and history teaching methods.

The evaluation was conducted with the server in the following setting: Virtuoso 6.1.6 on Ubuntu server 3.13 to manage SPARQL endpoints. The database was loaded with data dumps from DBpedia 3.9 and Freebase RDF of January 2015.

\section{$\S 2$ How are shallow questions evaluated?}

In the case of the shallow questions, the system generates them by using explicitly described semantic relation instances representing fact (predicate) knowledge. The size of a set of questions ( $\mathrm{R} \& \mathrm{C}$ based Question) generated by the system depends on the amount of semantic relation instances represented, although the system has an advantage of generating them adaptively to individual learners. Therefore, it is important to evaluate the coverage of human questions by the set of system generated questions to confirm whether the question generation function with current states of LOD can be available for building/assessing learners' fundamental knowledge. The evaluation of the shallow questions considered the 38 manually generated shallow questions about WWI (31: WWII) taken from the website SparkNotes. Each manually generated question was paired with an automatically generated question. An example of the process for the human generated question "Who won the Battle of the Falkland Islands?" (Answer: "United Kingdom") is as follows.

- First, we manually analyzed the human generated questions to identify the concept instances involved. The target concept instances of the question and answer were manually identified: "Battle of the Falkland Is- 
Table 1 Shallow Questions Evaluation Results

\begin{tabular}{c|c|cccc|c}
\hline \multicolumn{2}{c|}{ Mark } & $\mathbf{1}$ & $\mathbf{2}$ & $\mathbf{3}$ & $\mathbf{4}$ & Total \\
\hline \hline \multirow{2}{*}{$\begin{array}{c}\text { Number } \\
\text { of } \\
\text { couples }\end{array}$} & WWI & $5(13 \%)$ & $15(39 \%)$ & $14(37 \%)$ & $4(11 \%)$ & 38 \\
\cline { 2 - 7 } & WWII & $6(19 \%)$ & $15(48 \%)$ & $5(16 \%)$ & $5(16 \%)$ & 31 \\
\cline { 2 - 7 } & Total & $11(16 \%)$ & $30(43 \%)$ & $19(28 \%)$ & $9(13 \%)$ & 69 \\
\hline
\end{tabular}

lands" and "United Kingdom."

- Then, the system generated as many questions about the identified concepts as possible. The generated questions were about all the involved concept instances depending on the relation instance information available e.g. "Where did the Battle of the Falkland Islands happen?", or "Which battles involved United Kingdom?", etc.

- Finally, we manually selected the question with the closest possible meaning. In that case, the selected question was "What was the result of the Battle of the Falkland Islands?"

In total, 38 couples of questions about WWI (31: WWII) containing each one manually and one automatically generated question were created for the evaluation.

The evaluator was asked to give a mark from 1 to 4 for each couple, where 1 means the knowledge required to answer is different and 4 means the knowledge needed is the same. We asked the evaluator to set concrete criteria from the viewpoint of history learning.

\section{$\S 3$ How are deep questions evaluated?}

In the case of the deep questions, the knowledge that human and system can use to generate them is different.

On one side, a teacher has deep knowledge of the topic including the knowledge not written in a textbook as well as the ability to read between lines. Thus, a teacher can generate questions that require a learner to think between lines to construct historical deep understanding including knowledge not explicitly described.

On the other side, the system can generate only based on explicitly represented information and ontologies that require learners to state their opinion.

The purpose of evaluating the deep questions is to clarify whether the system can generate questions that trigger historically deep thinking by using explicitly represented domain knowledge in LOD and specific topic independent ontologies.

The evaluation considered the totality of the 20 (27: WWII) manually generated deep questions and 30 (30: WWII) deep questions automatically generated by the system. The automatically generated questions were generated by referring to the concept instances mentioned on the SparkNotes page "Key People and Terms" about WWI. Among 55 (75: WWII) concept instances identified, 30
(30: WWII) concept instances with the most semantic information were selected and used as a target for the question generation.

The manually and automatically generated questions were then scrambled together. The evaluator was provided with the resulting 50 (57: WWII) questions and instructed to judge the ability to deepen learners' understanding. Then, the evaluator was asked categorize all the questions into 5 categories (C1-C5). Questions in category C5 are good questions contributing to deepening the understanding of the learners, whereas questions in category $\mathrm{C} 1$ do not contribute significantly to deepening the understanding. The specific criteria were left up to the evaluator.

\section{$5 \cdot 2$ Results}

\section{$\S 1$ Quality of shallow questions}

Table 1 shows the number of couples for each mark. The evaluator described the criteria used for attributing grades during the evaluation as follows.

1: Both questions require different knowledge to be answered e.g. "To which other prominent leader was Kaiser Wilhelm II of Germany related?" (Human) and "Who is related to Wilhelm II, German Emperor?" (System).

2: Both questions focus on different parts of the target relation instance, but they require the same knowledge to be answered, e.g. "Who won the Battle of the Falkland Islands?" (Human) and "What was the result of the Battle of the Falkland Islands?" (System).

3: Both questions assess the same knowledge from different viewpoints (they require the same knowledge to be answered), e.g. "Who assumed power in Germany and led negotiations with the Allies after Wilhelm II lost power?" (Human) and "Who succeeded Wilhelm II, German Emperor?" (System).

4: Both questions have the same meaning e.g. "In what city was Archduke Franz Ferdinand assassinated?" (Human) and "Where did the Assassination of Archduke Franz Ferdinand of Austria take place?" (System).

The evaluator judged that couples marked 2, 3 and 4 require the same knowledge for junior and high school students to be answered. Thus, we recognize couples marked 2, 3 and 4 were the same questions from the viewpoint of requiring the same knowledge structure. In total, under the 
Table 2 Deep Questions Evaluation Results

\begin{tabular}{|c|c|c|c|c|c|c|c|}
\hline \multicolumn{2}{|c|}{ Category (Weight) } & \multirow{3}{*}{$\begin{array}{l}\text { C1 } \\
\text { (1) } \\
1\end{array}$} & \multirow{3}{*}{$\begin{array}{l}\text { C2 } \\
\text { (2) } \\
5\end{array}$} & \multirow{3}{*}{$\begin{array}{l}\text { C3 } \\
\text { (3) } \\
8\end{array}$} & \multirow{3}{*}{$\begin{array}{l}\text { C4 } \\
\text { (4) } \\
3\end{array}$} & \multirow{3}{*}{$\begin{array}{l}\text { C5 } \\
\text { (5) } \\
3\end{array}$} & \multirow{3}{*}{$\begin{array}{c}\text { W. Avg. } \\
3.1\end{array}$} \\
\hline & & & & & & & \\
\hline WWI & Human $(n=20)$ & & & & & & \\
\hline WWI & System $(n=30)$ & 1 & 1 & 19 & 9 & 0 & 3.2 \\
\hline \multirow{2}{*}{ WWII } & Human $(n=27)$ & 6 & 10 & 7 & 1 & 3 & 2.4 \\
\hline & System $(n=30)$ & 0 & 12 & 11 & 1 & 6 & 3.0 \\
\hline \multirow{2}{*}{ Total } & Human $(n=47)$ & $\overline{77}$ & $\overline{\overline{15}}$ & $\overline{15}$ & 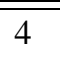 & 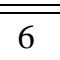 & 2.7 \\
\hline & System $(n=60)$ & 1 & 13 & 30 & 10 & 6 & 3.1 \\
\hline
\end{tabular}

conditions of the system, $84 \%$ of the manually generated questions about WWI and WWII could be covered by the system.

The remaining $16 \%$ of questions were not invalid or useless questions. For these questions, the system was not able to generate questions that require the same knowledge from the learners. The questions generated by the system asked about different basic knowledge.

During the interview after the above experiments, he suggested that even shallow questions for junior and high school students may become deep questions for university students with deep historical knowledge, because they interpret their questions differently. We understood this is a characteristic feature of history domain learning.

Thus, as an additional strict evaluation on the topic of WWI, evaluator was asked to judge for each couple about the quality of one question compared with the other. The results of this additional evaluation showed that for 39\% (15/38) of the couples, the questions did not have a difference in quality. The system generated question was better in $29 \%(11 / 38)$ of the couples, and the human generated one was better in $32 \%(12 / 38)$ of the couples. These results show no significant difference between the quality of the system and human generated shallow questions.

As a result, the question generation function has a potential to generate useful questions to construct learners' basic knowledge. Furthermore, it suggests that even shallow questions, in this case, $\mathrm{R} \& \mathrm{C}$ based question might be useful even for learners studying history in a university.

\section{$\S 2$ Quality of deep questions}

Table 2 shows the number of questions for each category. The result shows that it can generate questions of the same quality as the manually generated questions in average.

The criteria defined by the evaluator were:

C1) Questions asking facts.

C2) Questions asking causal relations.

C3) Others, more complex than $\mathrm{C} 2$ but does not require complete integrated knowledge like $\mathrm{C} 4$. It requires an understanding of the topic of the question and its context.

C4) Questions requiring integrated knowledge of the topic as a whole. It requires knowledge of the topic of the questions as well as a general understanding of the main topic important events and their context.

C5) Questions requiring a deep historical or political thinking. It requires having an understanding of global history and the relations between the topic and other historical topics.

The evaluator judged the quality of questions in category $\mathrm{C} 1$ and $\mathrm{C} 2$ are still meaningful, whereas the quality of the questions in category $\mathrm{C} 3$ or above are more suitable in a sense of prompting learners to build their own image of the past.

The system only uses information specific to one topic, i.e. using one of the key concepts defined in SparkNotes, to generate a question in this experiment. Although it depends on the templates that were used by the system, the majority of the questions are of reasonable quality ( $\mathrm{C} 3$ or above). Examples of questions for each category and for both WWI and WWII can be seen in Table 3.

As a result, the question generation function has a potential to generate useful questions to reinforce learners' deep understanding of historical topics.

\section{$5 \cdot 3$ Discussion}

The generated questions can cover most $(84 \%)$ of the shallow questions generated by humans. In addition, the deep questions generated by the system and humans have the same average quality. The results indicate that the questions generated by the system can be expected to support learners, in both acquiring basic knowledge and building a deep understanding, like human generated questions.

In addition, the system can generate a huge quantity of questions covering many historical topics. Because the generated questions are semantically correct and are meaningful for supporting learners, we consider that the HDQ Ontology built by specifying Graesser's taxonomy seems 
Table 3 Examples of deep questions

\begin{tabular}{|c|c|c|c|}
\hline \multirow{6}{*}{ 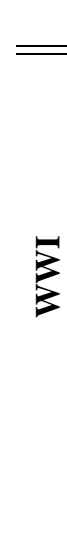 } & & Human & System \\
\hline & $\mathbf{C 1}$ & $\begin{array}{l}\text { What did Germany do in } 1917 \text { to hasten Russia's } \\
\text { exit from the war? }\end{array}$ & $\begin{array}{l}\text { Was the influence of George V important during } \\
\text { World War I? }\end{array}$ \\
\hline & $\mathbf{C 2}$ & $\begin{array}{l}\text { What was the initial purpose of Britain's invasion } \\
\text { of Mesopotamia? }\end{array}$ & $\begin{array}{l}\text { How did the fights of the First Battle of the Marne } \\
\text { change the course of World War I? }\end{array}$ \\
\hline & $\mathbf{C 3}$ & Why did Britain need control of the Dardanelles? & $\begin{array}{l}\text { What were the consequences of World War I for } \\
\text { the people living in Mons? }\end{array}$ \\
\hline & $\mathbf{C 4}$ & $\begin{array}{l}\text { What was the political result of Britain's invasion } \\
\text { of Gallipoli? }\end{array}$ & $\begin{array}{l}\text { How did the use of Submarine as mean of trans- } \\
\text { portation changed the course of World War I? }\end{array}$ \\
\hline & $\mathbf{C 5}$ & $\begin{array}{l}\text { World War I has often been described as an "un- } \\
\text { necessary war." Why? Do you agree? }\end{array}$ & - \\
\hline \multirow{5}{*}{ 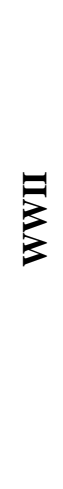 } & C1 & $\begin{array}{l}\text { What was the Soviet defense plan against Ger- } \\
\text { many? }\end{array}$ & - \\
\hline & C2 & $\begin{array}{l}\text { Was there any justification for Britain and } \\
\text { France's policy of appeasement? }\end{array}$ & $\begin{array}{l}\text { What were the consequences of World War II on } \\
\text { Nazi Germany? }\end{array}$ \\
\hline & $\mathbf{C 3}$ & $\begin{array}{l}\text { Why did Allied forces invade Italy after it had al- } \\
\text { ready surrendered? }\end{array}$ & $\begin{array}{l}\text { How did the fights of the Battle of Iwo Jima } \\
\text { change the course of World War II? }\end{array}$ \\
\hline & $\mathbf{C 4}$ & $\begin{array}{l}\text { What were Germany's mistakes in Russia and how } \\
\text { did they affect the outcome of the war? }\end{array}$ & $\begin{array}{l}\text { What kind of influence had Schutzstaffel on World } \\
\text { War II? }\end{array}$ \\
\hline & $\mathbf{C 5}$ & $\begin{array}{l}\text { Were Germany and Japan aggressions fundamen- } \\
\text { tally similar or fundamentally different? }\end{array}$ & $\begin{array}{l}\text { How would World War II have been different } \\
\text { without Adolf Hitler? }\end{array}$ \\
\hline
\end{tabular}

valid.

The main advantage of our question generation method for the self-directed learning system is that the questions are adapted to each learner. The system generates questions depending on the learners' knowledge states (represented by their concept map). The adaptability is beneficial to learners from the viewpoint of enhancing motivated learning.

From the technical viewpoint, our system has the advantage in a sense of generating deep questions based on the semantic processing (LOD). However, no existing system has been developed that can generate an answer to any history deep (essay) questions. Thus, it is important that we confirmed the deep questions generated with our method have a possibility of meaningfulness from the viewpoint of deepening historical understanding, even the system cannot give the answer.

One of the limitations of current LOD based question generation is that current LOD as a learning material of history is limited, i.e. the information described contains only facts and no opinions. Opinion information is included on Wikipedia as natural language but is not described on the current LOD.

An interesting issue, from the viewpoint of AI research, of applying the question generation function to the history domain is that it can generate valid and meaningful ques- tions based on the ontologies even if the answer is not explicitly represented in the LOD. For example, the answer to the question "What kind of influence had Schutzstaffel on World War II?", which is a category C4 question generated by the system, is not described in the LOD. A unique answer for this question does not exist as opposed to a question that could be asked in the mathematics domain. The evaluator (history professor) said "asking this type of question is meaningful in a sense of requiring integrated knowledge about the topic." It means that they contribute by encouraging thinking between lines apart from remembering facts. The reason why the system can generate this type of questions is that we could build reasonable ontologies as well as the domain of application being the history domain.

From this point of view, to answer C5 categorized deep questions such as "How would WWII have been different without Adolf Hitler?" or "Did technology fundamentally affect the outcome of the war? If so, how? If not, why not?" they need to understand the roles and influence of the events. Obviously, each of these questions does not have a unique correct answer but thinking by themselves about these kinds of questions in their internal selfconversation and thinking between the lines is important to build their own image of roles and states of persons, events and etc., even in the situation that the validity of 
the answer cannot be confirmed.

The results of both evaluations demonstrate the validity of our method for generating questions about the topics on 'World Wars'. However, the quality of the generated questions about other topics is not verified. We need to carefully address the quality of questions with the set of definitions specified in the question ontology. The results in other historical topics might be different: we might need to add definitions of history domain questions and natural language patterns in the question generation ontology to adapt, although our framework can accept such extensions without any changes of question generation system.

\section{Concluding Remarks and Future Works}

In this paper, we described a method for generating questions automatically by using LOD. The history domain ontology makes it possible to use the concept (relation) instances from two semantic resources (DBpedia and Freebase). The history dependent question ontology makes possible to generate content (topic) dependent questions using domain dependent but content (topic) independent question concept classes. One of the advantages of the system is that we can add definitions of history domain questions and natural language patterns in the question ontology without any changes of question generation system. The questions generated by the system can be expected to support learners in acquiring basic knowledge and deepening their understanding of history.

The evaluation showed that the system could generate good quality questions about 'World Wars' by using the current LOD. The experimental results showed that the questions generated by the system can cover the majority of the questions generated by humans. In addition, the questions enhancing history thinking generated by the system and by human were of the same average quality. By considering the system can generate much more questions not appearing on SparkNotes and its adaptability, the results described in this paper seems quite meaningful in the situation of individual learners' support. However, we recognized there is no unique criterion to evaluate the quality of history questions. More investigation has to be done by other history professors.

In future work, by developing a richer ontology including multiple concepts and relations, and with richer semantics being embedded in LOD, the system could become able to generate more meaningful questions from richer relation instances information. Furthermore, we need to carefully address the quality of questions about other topics with a combination of a set of definitions spec- ified in question ontology and the topic.

In this paper, we build a domain independent general framework for question generation using LOD, although we confirmed that the quality of questions generated by the method only on the topic of World Wars. The important point to realize the generality of the system is we separate domain independent and history dependent concepts of the question generation ontology. Because the meaningful questions to enhance understanding depend on respective learning domains, we should systemize domain dependent question ontology to adapt to other domains. In other words, the system has a possibility to adapt to other domains by changing the history dependent question ontology to another domain dependent one.

Furthermore, we think two requirements need to be satisfied for the question generation to adapt to other domains:

1. Meaningful information needs to be available on the LOD to be able to support learning about topics in the targeted domain.

2. The targeted domain ontology must be specified. The current ontology only specifies concept and relation classes for the history domain.

The kind of learning domains that can satisfy above requirements should be clarified in the future papers.

Finally, the way the function is embedded in the system and its utility from the viewpoint of learning support should be carefully addressed through our next works, e.g. how we embed the question generation function into the system, how and when the system shows shallow and deep questions, how many questions the system shows, whether the system can give positive influence to learners, and so on. We expect to conduct several experiments to address the above points in future works.

\section{Acknowledgments}

We would like to offer our special thanks to Professor Akifumi Sumitomo for his meaningful comments.

This work was supported by JSPS KAKENHI Grant Numbers 24300288, 15H02934.

\section{References $\diamond$}

[01] Agarwal, M., Shah, R. and Mannem, P. (2011). Automatic question generation using discourse cues. In Proceedings of the 6th Workshop on Innovative Use of NLP for Building Educational Applications, p. 19. Association for Computational Linguistics.

[02] Biswas, G., Roscoe, R., Jeong, H. and Sulcer, B. (2009). Promoting self-regulated learning skills in agent-based learning environments. In Proceedings of the 17th International Conference on Computers in Education, pp. 67-74.

[03] Bizer, C., Lehmann, J., Kobilarov, G., Auer, S., Becker, C., Cyganiak, R. and Hellmann, S. (2009). DBpedia-A crystallization point 
for the Web of Data. In Web Semantics: Science, Services and Agents on the World Wide Web, Vol.7, No.3, pp. 154-165.

[04] Bollacker, K., Evans, C., Paritosh, P., Sturge, T. and Taylor, J. (2008). Freebase: a collaboratively created graph database for structuring human knowledge. In Proceedings of the 2008 ACM SIGMOD International Conference on Management of Data, pp. 1247-1250.

[05] Bransford, J. D., Brown, A. and Cocking, R. (1999). How People Learn: Mind, Brain, Experience, and School. Washington, DC: National Research Council.

[06] De Jong, T. (2006). Technological advances in inquiry learning. In Science, Vol.312, No.5773, pp. 532-533.

[07] Graesser, A., Ozuru, Y. and Sullins, J. (2010). What is a good question?. In Bringing Reading Research to Life. Guilford Press.

[08] Heilman, M. and Smith, N.A. (2009). Question Generation via Overgeneration Transformations and Ranking, No. CMU-LTI-09013. Carnegie-Mellon University, Pittsburgh.

[09] Hirashima, T., Yamasaki, K., Fukuda, H. and Funaoi, H. (2011). Kit-build concept map for automatic diagnosis. In Artificial Intelligence in Education, pp. 466-468. Springer Berlin Heidelberg.

[10] Hmelo-Silver, C. E., Duncan, R. G. and Chinn, C. A. (2007). Scaffolding and achievement in problem-based and inquiry learning: A response to Kirschner, Sweller, and Clark (2006). In Educational Psychologist, Vol.42, No.2, pp. 99-107.

[11] Husbands, C. (1996). What is History Teaching?: Language, Ideas and Meaning in Learning about the Past. Berkshire: Open University Press.

[12] Husbands, C., Kitson, A. and Pendry, A. (2003). Understanding History Teaching: Teaching and Learning about the Past in Secondary Schools. McGraw-Hill International.

[13] Jouault, C. and Seta, K. (2013). Adaptive self-directed learning support by question generation in a semantic open learning space. In International Journal of Knowledge and Web Intelligence, Vol.4, No.4, pp. 349-363.

[14] Jouault, C. and Seta, K. (2013). Wikipedia-based concept-map building and question generation. In The Journal of Information and Systems in Education, Vol.12, No.1, pp. 50-55.

[15] Jouault, C. and Seta, K. (2014). Content-dependent question generation for history learning in semantic open learning space. In Intelligent Tutoring Systems, pp. 300-305. Springer International Publishing.

[16] Kashihara, A. and Taira, K. (2009). Developing navigation planning skill with learner-adaptable scaffolding. In Proceedings of the 2009 Conference on Artificial Intelligence in Education: Building Learning Systems that Care: From Knowledge Representation to Affective Modelling, pp. 433-440. IOS Press.

[17] Le, N.T. and Pinkwart, N. (2014). Question Generation Using WordNet. In Proceedings of the 22nd International Conference on Computers in Education.

[18] Li, H., Duan, Y., Clewley, D. N., Morgan, B., Graesser, A. C., Shaffer, D. W. and Saucerman, J. (2014). Question asking during collaborative problem solving in an online game environment. In Intelligent Tutoring Systems, pp. 617-618. Springer International Publishing.

[19] Lindberg, D., Popowich, F., Nesbit, J. and Winne, P. (2013). Generating natural language questions to support learning on-line. In Proceedings of the 14th European Workshop NLG, pp. 105-114. Association for Computational Linguistics.

[20] Mazidi, K. and Nielsen, R. D. (2014). Pedagogical evaluation of automatically generated questions. In Intelligent Tutoring Systems, pp. 294-299. Springer International Publishing.

[21] Nesbit, J.C. and Adesope, O. O. (2006) Learning with concept and knowledge maps: A meta-analysis. In Review of Educational Research, Vol. 76, No.3, pp. 413-448.

[22] Otero, J. (2009). Question generation and anomaly detection in texts. Handbook of Metacognition in Education, pp. 47-59.

[23] Riley, M. (2000). Into the Key Stage 3 history garden: choosing and planting your enquiry questions. In Teaching History. London: Historical Association.

[24] Roth, W. M. (1996). Teacher questioning in an open-inquiry learning environment: Interactions of context, content, and student responses. Journal of Research in Science Teaching, Vol.33, No.7, pp. 709-736.

[25] Seta, K., Noguchi, D. and Ikeda, M. (2011). Presentation-based collaborative learning support system to facilitate meta-cognitively aware learning communication. In The Journal of Information and Systems in Education, Vol.9, No.1, pp.3-14.

[26] Seta, K. and Ikeda, M. (2011). Presentation based meta-learning environment by facilitating thinking between lines: A model based approach. In Toyohide Watanabe and Lakhmi C. Jain (Eds.): Innovations in Intelligent Machines-2-Intelligent Paradigms and Applications, Studies in Computational Intelligence, Vol. 376, pp. 143-166. Springer.

[27] Slotta, J. D. (2004). The web-based inquiry science environment (WISE): Scaffolding knowledge integration in the science classroom. In Internet Environments for Science Education, pp. 203-232.

[28] Smart, L. (1996). Using I.T. in Primary School History. London: Cassell.

[29] SparkNotes Editors (accessed December 12, 2014). SparkNote on World War I (1914-1919). SparkNotes LLC. 2005. http://www.sparknotes.com/history/european/ww1/

[30] Stow, W. and Haydn, T. (2000). 7 Issues in the teaching of chronology. In Issues in History Teaching, p. 83. Routledge.

[31] Thuering, M., Hannemann, J., and Haake, J. M. (1995). Hypermedia and cognition: Designing for comprehension, Communication of the ACM, Vol.38, No.8, pp. 57-66.

[32] Wolfe, J.H. (1976). Automatic question generation from text. ACM SIGCUE Outlook 10(SI), pp. 104-112.

[33] Wyse, B. and Piwek, P. (2009). Generating questions from openlearn study units. In AIED 2009 Workshop Proceedings Volume 1: The 2nd Workshop on Question Generation.

[34] Zimmerman, B. J. and Schunk, D. H. (Eds.). (2001). SelfRegulated Learning and Academic Achievement: Theoretical Perspectives. Routledge.

\section{〔担当委員 : 川村 隆浩〕}

Received February 09, 2015.

\section{Author's Profile}

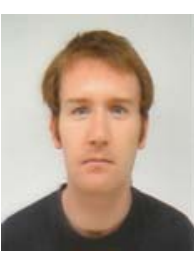

\section{Corentin Jouault}

Corentin Jouault received the M.E. in Computer Science from EISTI, France in 2010. He is currently a Ph.D. candidate at the Graduate School of Science, Osaka Prefecture University. His research interests include ontological engineering, semantic web, artificial intelligence, intelligent tutoring systems and software engineering. He is a member of APSCE.

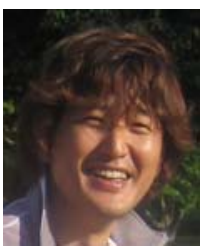

\section{Kazuhisa Seta (Member)}

Kazuhisa Seta received a B.E. and M.E. from Ryukoku University in 1993 and 1995, respectively. He received a Ph.D. from Osaka University in 1998. He is currently a professor in the College of Sustainable System Sciences and the Graduate School of Science, Osaka Prefecture University. His research interests include software engineering, intelligent tutoring systems, human resource management, and ontological engineering. He received a Best Paper Award from the Japanese Society for Information and Systems in Education in 2012 and 2015. He is a member of IEICE, IPSJ, JSiSE, JCSS, APSCE, and IAIED.

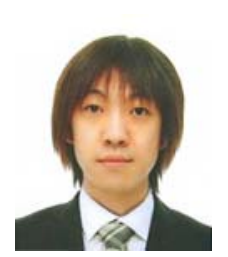

\section{Yuki Hayashi (Member)}

Yuki Hayashi received his B.E., M.E., and Ph.D. from Nagoya University in 2007, 2009, and 2012, respectively. From 2012 to 2014, he was an assistant professor in Seikei University. He is currently an assistant professor in the College of Sustainable System Sciences, Osaka Prefecture University. His research interests include computer-supported collaborative learning and human-computer interaction. He is a member of IPSJ, JSiSE, and HIS. 